\title{
The Dynamics of an Impulsive Predator-Prey System with Stage Structure and Holling Type III Functional Response
}

\author{
Zhixiang Ju, ${ }^{1}$ Yuanfu Shao, ${ }^{1}$ Xiaolan Xie, ${ }^{2}$ Xiangmin Ma, ${ }^{1}$ and Xianjia Fang ${ }^{1}$ \\ ${ }^{1}$ School of Science, Guilin University of Technology, Guilin, Guangxi 541004, China \\ ${ }^{2}$ Institute of Information Science and Technology, Guilin University of Technology, Guilin, Guangxi 541004, China \\ Correspondence should be addressed to Yuanfu Shao; shaoyuanfu@163.com
}

Received 7 May 2014; Revised 12 September 2014; Accepted 14 September 2014

Academic Editor: Yonghui Xia

Copyright (c) 2015 Zhixiang Ju et al. This is an open access article distributed under the Creative Commons Attribution License, which permits unrestricted use, distribution, and reproduction in any medium, provided the original work is properly cited.

\begin{abstract}
Based on the biological resource management of natural resources, a stage-structured predator-prey model with Holling type III functional response, birth pulse, and impulsive harvesting at different moments is proposed in this paper. By applying comparison theorem and some analysis techniques, the global attractivity of predator-extinction periodic solution and the permanence of this system are studied. At last, examples and numerical simulations are given to verify the validity of the main results.
\end{abstract}

\section{Introduction}

In recent decades, with the increase of population and the development of science and technology, human accelerated the exploitation of natural resources. Many harvesting predator-prey models have been studied. The dynamic relationship between predators and their preys has long been and will continue to be one of the dominant themes in both ecology and mathematical ecology due to its universal existence and importance; see [1-6] and the references cited therein. On the other hand, in the real world, many species usually go through two or even more life stages as they proceed from birth to death. Thus, it is practical to introduce the stage structure into predator-prey models; see [7-12]. For example, Wei and Wang [13] considered the following predator-prey system with stage structure:

$$
\begin{aligned}
\dot{x}(t) & =x(t)\left(r_{1}-a_{11} x(t)-a_{12} y_{2}(t)\right), \\
\dot{y}_{1}(t) & =\alpha x(t) y_{2}(t)-\gamma y_{1}(t)-\alpha e^{-\gamma \tau} x(t-\tau) y_{2}(t-\tau), \\
\dot{y}_{2}(t) & =\alpha e^{-\gamma \tau} x(t-\tau) y_{2}(t-\tau)-r_{2} y_{2}(t)-a_{22} y_{2}^{2}(t),
\end{aligned}
$$

where $x(t), y_{1}(t)$, and $y_{2}(t)$ denote the densities of prey population and immature and mature individual predators at time $t$, respectively. The meanings of all parameters may refer to [13]. Authors obtained the sufficient conditions of the persistence for system (1).

However, it is well known that many evolution processes are characterized by the fact that at certain moments their stage changes abruptly. For example, for IPM strategy on ecosystem, the predators are released periodically every time $T$, and periodic catching or spraying pesticide is also applied. Hence, the predator and prey experience a change of state abruptly. Consequently, it is natural to assume that these processes act in the form of impulse. Impulsive methods have been applied in almost every field of applied sciences. For example, many population models assume that the populations are born throughout the year, whereas it is often the case that many species give birth only during a single period of the year; that is, births occur in regular pulses. Hence, the authors Z. Xiang, D. Long, and X. Y. Song gave a single population logistic model with birth pulse and impulsive harvesting at different moments as follows:

$$
\begin{aligned}
\dot{x}(t) & =-\mu x(t), \quad t \neq(n+l) T, t \neq(n+1) T, \\
\Delta x(t) & =r x(t)\left(1-\frac{x(t)}{K}\right), \quad t=(n+l) T, \\
\Delta x(t) & =-p x(t), \quad t=(n+1) T, n \in Z^{+},
\end{aligned}
$$

where $x(t)$ represents the density of the resource population at time $t$. Parameter $r$ is the intrinsic growth rate, the positive 
constant $K$ is referred to as the environmental carrying capacity, and parameter $\mu$ is the death rate of resource population. Parameter $p$ denotes the harvest rate of resource population. For more details of the biological meaning of system (2), we can refer to [14-17].

In addition, in the description of dynamical interactions on predators and their preys, a crucial element of all models is the classic definition of a predator's functional response. A functional response of the predator to the prey density refers to the change in the density of prey attached per unit time per predator as the prey density changes. For example, Zhang et al. [18] suggested a function

$$
\varphi(x)=\frac{x^{2}}{c+x^{2}},
$$

called Holling type III. It is monotonic in the first quadrant, that is, if the prey population increases, then the consumption rate of prey per predator will increase too. And $\sqrt{c}$ is the halfsaturation constant. The field of research on the dynamics of impulsive predator-prey model with functional response seems to be a new increasingly interesting area, which draws many scholars' attention.

Moreover, by the picture of ocean's food-chain, we know that small fish can prey on fish larvae as a predator; also it can be eaten by the higher predator as a prey (see [19]). Hence, according to the nature of biological resource management, it is interesting to investigate impulsive harvesting on prey and mature predator (e.g., the small fish and higher predator in the ocean's food-chain) simultaneously at some fixed time.

Based on the above discussion, we consider the stagestructured predator-prey model with Holling type III functional response, birth pulse, and impulsive harvesting at different moments as follows:

$$
\begin{aligned}
\dot{x}(t)= & -\beta \frac{x^{2}(t)}{c+x^{2}(t)} y_{2}(t)-d_{1} x(t), \\
\dot{y}_{1}(t)= & \lambda \beta \frac{x^{2}(t)}{c+x^{2}(t)} y_{2}(t) \\
& -\lambda \beta e^{-d_{2} \tau} \frac{x^{2}(t-\tau)}{c+x^{2}(t-\tau)} y_{2}(t-\tau)-d_{2} y_{1}(t), \\
\dot{y}_{2}(t)= & \lambda \beta e^{-d_{2} \tau} \frac{x^{2}(t-\tau)}{c+x^{2}(t-\tau)} y_{2}(t-\tau) \\
& -d_{3} y_{2}(t)-d_{4} y_{2}^{2}(t), \\
x\left(t^{+}\right)= & x(t)+r x(t)\left(1-\frac{x(t)}{K}\right), \\
y_{1}\left(t^{+}\right)= & y_{1}(t), \quad t=n T, \quad t \neq n T, \\
y_{2}\left(t^{+}\right)= & y_{2}(t), \quad t=(n+l-1) T, \\
x\left(t^{+}\right)= & \left(1-p_{1}\right) x(t), \\
y_{1}\left(t^{+}\right)= & y_{1}(t), \\
y_{2}\left(t^{+}\right)= & \left(1-p_{2}\right) y_{2}(t), \\
&
\end{aligned}
$$

where $x(t)$ denotes the density of the prey and $y_{1}(t), y_{2}(t)$ represent the immature and mature predator densities, respectively. Parameters $r, K, \beta, \lambda, d_{1}, d_{2}, d_{3}$, and $d_{4}$ are positive constants, where $r$ is the intrinsic growth rate of the prey, $K$ denotes the capacity rate, concerned with the maintaining of the evolution of the population, $\beta$ represents the predation rate of predator, $\lambda$ is the conversion rate that translated into predator population increase, $d_{1}, d_{2}$, and $d_{3}$ denote the death rate of prey, immature predator, and mature predator, respectively, and $d_{4}$ is the intrinsicspecific competition rate of the mature predator. Parameter $\tau$ represents a constant time from immaturity to maturity. Parameters $p_{1}, p_{2}$ denote the harvesting rates of prey and mature predator at $t=n T, n \in Z_{+}$, respectively, $Z_{+}=$ $\{1,2, \ldots\}$, and $T$ is the period of the impulsive effect.

It is well known that, in the sustainable development of natural resources, it is very important to study the sustainable survival of species. So, in this paper, we aim to investigate the global attractivity of predator-extinction periodic solution and the permanence of system (4). From the biological point of view, we only consider (4) in the biological meaning region

$$
D=\left\{\left(x(t), y_{1}(t), y_{2}(t)\right): x(t) \geq 0, y_{1}(t) \geq 0, y_{2}(t) \geq 0\right\} \text {. }
$$

This paper is organized as follows. Firstly, some preliminaries are given in Section 2. In Section 3, the sufficient conditions for the global attractivity of predator-extinction periodic solution are obtained. The permanence of system (4) is investigated in Section 4. In Section 5, we present some examples and simulations to illustrate our results. At last, a brief conclusion is given in Section 6.

\section{Preliminaries}

In this section, some definitions and lemmas are introduced.

Let $R_{+}=[0, \infty)$ and $R_{+}^{3}=\left\{x \in R^{3}, x \geq 0\right\}$. Denote $f=$ $\left(f_{1}, f_{2}, f_{3}\right)^{T}$ to be the map defined by the right hand of system (4). Let $V: R_{+} \times R_{+}^{3} \rightarrow R_{+}$; then $V$ is said to belong to class $V_{0}$, if

(i) $V$ is continuous in $((n-1) T,(n+l-1) T] \times R_{+}^{3}$ and $((n+l-1) T, n T] \times R_{+}^{3}$ for each $x \in R_{+}^{3}, n \in N$, $\lim _{(t, y) \rightarrow\left((n+l-1) T^{+}, x\right)} V(t, y)=V\left((n+l-1) T^{+}, x\right)$ and $\lim _{(t, y) \rightarrow\left(n T^{+}, x\right)} V(t, y)=V\left(n T^{+}, x\right)$ exists,

(ii) $V$ is locally Lipschitzian in $x$.

Definition 1. Let $V \in V_{0}$, and $(t, v) \in((n-1) T,(n+l-1) T] \times R_{+}^{3}$ and $((n+l-1) T, n T] \times R_{+}^{3}$, the upper right derivative of $V(t, v)$ with respect to the impulsive differential system (4) is defined as

$$
D^{+} V(t, v)=\lim _{h \rightarrow 0^{+}} \sup \frac{1}{h}[V(t+h, v+h f(t, v))-V(t, v)] .
$$


The solution of (4), denote by $v=\left(x(t), y_{1}(t), y_{2}(t)\right)^{T}$, is a piecewise continuous function $v: R_{+} \rightarrow R_{+}^{3}, v(t)$ is continuous on $((n-1) T,(n+l-1) T] \times R_{+}^{3}$ and

$$
((n+l-1) T, n T] \times R_{+}^{3} \quad(n \in N, 0 \leq l \leq 1) .
$$

Obviously the existence and uniqueness of solutions of (4) is guaranteed by the smoothness properties of $f$, which denotes the map defined by the right hand of system (4).

The following lemmas are useful for the proof of the main results.

Lemma 2 (see $[5,7])$. Consider the following differential equation:

$$
\dot{x}(t)=a x(t-\tau)-b x(t)-c x^{2}(t),
$$

where $a, b, c$, and $\tau$ are positive constants and $x(t)>0$ for $t \in[-\tau, 0]$. We have the following:

(i) if $a<b$, then $\lim _{t \rightarrow+\infty} x(t)=0$;

(ii) if $a>b$, then $\lim _{t \rightarrow+\infty} x(t)=(a-b) / c$.

Lemma 3 (see [14]). Consider the following system:

$$
\begin{aligned}
\dot{u}(t) & =-w u(t), \quad t \neq(n+l-1) T, t \neq n T, \\
u\left(t^{+}\right) & =u(t)+r u(t)\left(1-\frac{u(t)}{K}\right), \quad t=(n+l-1) T, \\
u\left(t^{+}\right) & =(1-p) u(t), \quad t=n T .
\end{aligned}
$$

Then, system (9) has a positive periodic solution $u^{*}(t)$ with period $T$, which is globally stable, where

$$
u^{*}(t)=\left\{\begin{array}{c}
e^{w l T}\left(\frac{1+r}{r} K-\frac{e^{w T}}{(1-p) r} K\right) e^{-w(t-(n-1) T)}, \\
t \in((n-1) T,(n+l-1) T], \\
\frac{e^{w T}}{1-p}\left(\frac{1+r}{r} K-\frac{e^{w T}}{(1-p) r} K\right) e^{-w(t-(n+l-1) T),} \\
t \in((n+l-1) T, n T],
\end{array}\right.
$$

if $p<1-\left(e^{w T} /(1+r)\right)$.

Lemma 4. There is a positive constant $M$ such that $x(t) \leq$ $M / \lambda, y_{1}(t) \leq M$, and $y_{2}(t) \leq M$ for every solution $\left(x(t), y_{1}(t)\right.$, $\left.y_{2}(t)\right)$ of system (4) with all $t$ sufficiently large, where $\lambda$ is a positive constant defined in system (4).

Proof. Define $V(t)=\lambda x(t)+y_{1}(t)+y_{2}(t)$.

If $t \neq(n+l-1) T, t \neq n T$, we let $d=\min \left\{d_{1}, d_{2}, d_{3}\right\}$; then

$$
\begin{aligned}
D^{+} V(t)+d V(t)= & -\lambda\left(d_{1}-d\right) x(t)-\left(d_{2}-d\right) y_{1}(t) \\
& -\left(d_{3}-d\right) y_{2}(t)-d_{4} y_{2}^{2}(t) \leq 0,
\end{aligned}
$$

If $t=(n+l-1) T$, we have

$$
\begin{aligned}
V\left((n+l-1) T^{+}\right) & =V((n+l-1) T)+\lambda r x(t)\left(1-\frac{x(t)}{K}\right) \\
& \leq V((n+l-1) T)+\frac{\lambda r K}{4},
\end{aligned}
$$

and if $t=n T$, it is clear that $V\left(n T^{+}\right) \leq V(n T)$.

Then, by Lemma 2.2 of [20], for all $t \geq 0$, we have

$$
\begin{aligned}
V(t) \leq & V\left(0^{+}\right) e^{-d_{1} t}+\frac{\lambda r K}{4} \frac{e^{-d(t-T)}}{1-e^{-d T}} \\
& +\frac{\lambda r K}{4} \frac{e^{d T}}{e^{d T}-1} \longrightarrow \frac{\lambda r K}{4} \frac{e^{d T}}{e^{d T}-1}, \quad t \longrightarrow \infty .
\end{aligned}
$$

Therefore, there exists a positive constant

$$
M=\frac{\lambda r K}{4} \frac{e^{d T}}{e^{d T}-1}
$$

such that $x(t) \leq M / \lambda, y_{1}(t) \leq M$, and $y_{2}(t) \leq M$ for $t$ large enough. This completes the proof.

\section{Global Attractivity of a Predator-Extinction Periodic Solution}

In this section, we will demonstrate the existence and global attractivity of a predator-extinction periodic solution, in which the predator individuals are entirely absent from the population permanently; that is, $y_{1}(t)=0$ and $y_{2}(t)=0$ for all $t \geq 0$.

Firstly, by Lemma 3, we can easily obtain the existence of predator-extinction periodic solution for system (4).

Theorem 5. System (4) has a predator-extinction periodic solution $\left(x^{*}(t), 0,0\right)$ which is globally stable; that is, for $t \in$ $((n-1) T, n T]$ and any solution $\left(x(t), y_{1}(t), y_{2}(t)\right)$ of system $(4)$, we have $x(t) \rightarrow x^{*}(t)$ as $t \rightarrow \infty$, where

$$
x^{*}(t)=\left\{\begin{array}{c}
e^{d_{1} l T}\left(\frac{1+r}{r} K-\frac{e^{d_{1} T}}{\left(1-p_{1}\right) r} K\right) e^{-d_{1}(t-(n-1) T),} \\
t \in((n-1) T,(n+l-1) T], \\
\frac{e^{d_{1} T}}{1-p_{1}}\left(\frac{1+r}{r} K-\frac{e^{d_{1} T}}{\left(1-p_{1}\right) r} K\right) e^{-d_{1}(t-(n+l-1) T),} \\
t \in((n+l-1) T, n T],
\end{array}\right.
$$

and $x^{*}\left(0^{+}\right)=e^{d_{1} l T}\left(((1+r) / r) K-\left(e^{d_{1} T} /\left(1-p_{1}\right) r\right) K\right)$.

Next, we give the conditions on the global attractivity of the predator-extinction periodic solution $\left(x^{*}(t), 0,0\right)$ of the system (4).

Theorem 6. The predator-extinction periodic solution $\left(x^{*}(t)\right.$, $0,0)$ of system (4) is globally attractive, if

$$
\begin{aligned}
& \left(\mathrm{A}_{1}\right) 1-p_{1}-\left(e^{d_{1} T} /(1+r)\right)>0, \\
& \left(\mathrm{~A}_{2}\right) \lambda \beta e^{-d_{2} \tau}\left(\rho^{2} /\left(c+\rho^{2}\right)\right)<d_{3} .
\end{aligned}
$$


Proof. Let $\left(x(t), y_{1}(t), y_{2}(t)\right)$ be any solution of system (4); from the first, the fourth, and the seventh equations of system (4), we have

$$
\begin{aligned}
\dot{x}(t) & \leq-d_{1} x(t), \quad t \neq(n+l-1) T, t \neq n T, \\
x\left(t^{+}\right) & =x(t)+r x(t)\left(1-\frac{x(t)}{K}\right), \quad t=(n+l-1) T, \\
x\left(t^{+}\right) & =\left(1-p_{1}\right) x(t), \quad t=n T .
\end{aligned}
$$

Consider the auxiliary system of (16) as follows:

$$
\begin{aligned}
\dot{z}_{1}(t) & =-d_{1} z_{1}(t), \quad t \neq(n+l-1) T, t \neq n T, \\
z_{1}\left(t^{+}\right) & =z_{1}(t)+r z_{1}(t)\left(1-\frac{z_{1}(t)}{K}\right), \quad t=(n+l-1) T, \\
z_{1}\left(t^{+}\right) & =\left(1-p_{1}\right) z_{1}(t), \quad t=n T .
\end{aligned}
$$

By Lemma 3 and condition $\left(A_{1}\right)$, we have

$$
z_{1}^{*}(t)=\left\{\begin{array}{c}
e^{d_{1} l T}\left(\frac{1+r}{r} K-\frac{e^{d_{1} T}}{\left(1-p_{1}\right) r} K\right) e^{-d_{1}(t-(n-1) T)}, \\
t \in((n-1) T,(n+l-1) T], \\
\frac{e^{d_{1} T}}{1-p_{1}}\left(\frac{1+r}{r} K-\frac{e^{d_{1} T}}{\left(1-p_{1}\right) r} K\right) e^{-d_{1}(t-(n+l-1) T)}, \\
t \in((n+l-1) T, n T],
\end{array}\right.
$$

which is unique and positive periodic solution of system (17) and is globally attractive. Applying comparison theorem of impulsive differential equation [21], there exist $n_{1} \in Z_{+}$and a sufficiently small constant $\varepsilon_{1}>0$ such that

$$
\begin{aligned}
x(t) & \leq z_{1}(t) \leq z_{1}^{*}(t)+\varepsilon_{1} \\
& \leq \frac{e^{d_{1} T}}{1-p_{1}}\left(\frac{1+r}{r} K-\frac{e^{d_{1} T}}{\left(1-p_{1}\right) r} K\right)+\varepsilon_{1} \stackrel{\Delta}{=} \rho,
\end{aligned}
$$

for $t \geq\left(n_{1}-1\right) T$.

From inequality (19), we can obtain

$$
\begin{array}{r}
\dot{y}_{2}(t) \leq \lambda \beta e^{-d_{2} \tau} \frac{\rho^{2}}{c+\rho^{2}} y_{2}(t-\tau)-d_{3} y_{2}(t)-d_{4} y_{2}^{2}(t), \\
t \neq n T, \\
y_{2}\left(t^{+}\right)=\left(1-p_{2}\right) y_{2}(t) \leq y_{2}(t), \quad t=n T .
\end{array}
$$

Consider the auxiliary system of (20):

$$
\dot{z}_{2}(t)=\lambda \beta e^{-d_{2} \tau} \frac{\rho^{2}}{c+\rho^{2}} z_{2}(t-\tau)-d_{3} z_{2}(t)-d_{4} z_{2}^{2}(t) .
$$

Applying Lemma 2, $\left(\mathrm{A}_{2}\right)$, and comparison theorem, we have $z_{2}(t) \rightarrow 0$ as $t \rightarrow \infty$. Then, for any small constant $\varepsilon_{2}>0$, there exists $t_{1}>0$, such that

$$
y_{2}(t) \leq z_{2}(t) \leq \varepsilon_{2}, \quad t>t_{1} .
$$

By the second equation of system (4) and (22), we have

$$
\dot{y}_{1}(t) \leq \lambda \beta \frac{\rho^{2}}{c+\rho^{2}} \varepsilon_{2}-d_{2} y_{1}(t) .
$$

Consider the auxiliary system of (23) as follows:

$$
\dot{z}_{3}(t)=\lambda \beta \frac{\rho^{2}}{c+\rho^{2}} \varepsilon_{2}-d_{2} z_{3}(t) .
$$

Integrating (24), one can easily get $z_{3}(t)=\lambda \beta\left(\rho^{2} / d_{2}(c+\right.$ $\left.\left.\rho^{2}\right)\right) \varepsilon_{2}$ as $t \rightarrow \infty$. Then, for any small constant $\varepsilon_{3}>0$, there exists $t_{2}>0$ such that

$$
y_{1}(t) \leq z_{3}(t) \leq \lambda \beta \frac{\rho^{2}}{d_{2}\left(c+\rho^{2}\right)} \varepsilon_{2}+\varepsilon_{3}, \quad t>t_{2} .
$$

According to the first, the fourth, and the seventh equations of (4), we have

$$
\begin{aligned}
\dot{x}(t) & \geq-\left(\beta \xi \varepsilon_{2}+d_{1}\right) x(t), \quad t \neq(n+l-1) T, t \neq n T, \\
x\left(t^{+}\right) & =x(t)+r x(t)\left(1-\frac{x(t)}{K}\right), \quad t=(n+l-1) T, \\
x\left(t^{+}\right) & =\left(1-p_{1}\right) x(t), \quad t=n T,
\end{aligned}
$$

where $\xi=\max \left\{\sqrt{c} / 2 c, \rho /\left(c+\rho^{2}\right)\right\}$. Again, consider the auxiliary system of (26):

$$
\begin{aligned}
\dot{z}_{4}(t) & =-d_{1} z_{4}(t), \quad t \neq(n+l-1) T, t \neq n T, \\
z_{4}\left(t^{+}\right) & =z_{4}(t)+r z_{4}(t)\left(1-\frac{z_{4}(t)}{K}\right), \quad t=(n+l-1) T, \\
z_{4}\left(t^{+}\right) & =\left(1-p_{1}\right) z_{4}(t), \quad t=n T .
\end{aligned}
$$

In view of Lemma 3, system (27) has a unique positive periodic solution as follows:

$$
z_{4}^{*}(t)=\left\{\begin{array}{c}
e^{\left(\beta \xi \varepsilon_{2}+d_{1}\right) l T}\left(\frac{1+r}{r} K-\frac{e^{\left(\beta \xi \varepsilon_{2}+d_{1}\right) T}}{\left(1-p_{1}\right) r} K\right) \\
\times e^{-\left(\beta \xi \varepsilon_{2}+d_{1}\right)(t-(n-1) T)}, \\
t \in((n-1) T,(n+l-1) T], \\
\frac{e^{\left(\beta \xi \varepsilon_{2}+d_{1}\right) T}}{1-p_{1}}\left(\frac{1+r}{r} K-\frac{e^{\left(\beta \xi \varepsilon_{2}+d_{1}\right) T}}{\left(1-p_{1}\right) r} K\right) \\
\times e^{-\left(\beta \xi \varepsilon_{2}+d_{1}\right)(t-(n+l-1) T)}, \\
t \in((n+l-1) T, n T] .
\end{array}\right.
$$

Similarly, for any arbitrary small constant $\varepsilon_{1}>0$, there exists $t_{3}>0$ such that $x(t) \geq z_{4}^{*}(t)-\varepsilon_{1} \stackrel{\Delta}{=} \delta$. Let $\varepsilon_{2} \rightarrow 0$; then, $z_{4}^{*}(t) \rightarrow x^{*}(t)$, and

$$
x(t) \geq x^{*}(t)-\varepsilon_{1} .
$$

It follows from (29) and (19) that $x(t) \rightarrow x^{*}(t)$ as $t \rightarrow \infty$. Let $\varepsilon_{2}, \varepsilon_{3} \rightarrow 0$; then, we have $y_{1}(t) \rightarrow 0, y_{2}(t) \rightarrow 0$ as $t$ sufficiently large enough. The proof is complete.

\section{Permanence of System (4)}

In the real world, from the principle of ecosystem balance and saving resources, we only need to control the predator under 
the economic threshold level and not to eradicate the predator totally. Thus, we focus on the permanence of system (4).

First, we give the definition of permanence.

Definition 7. System (4) is said to be persistent if there exist positive constants $m$ and $M$ such that every positive solution $\left(x(t), y_{1}(t), y_{2}(t)\right)$ of system (4) satisfies $m \leq$ $x(t), y_{1}(t), y_{2}(t) \leq M$ for $t$ sufficiently large enough.

Theorem 8. System (4) is permanent, if the following conditions hold:

$$
\begin{aligned}
& \left(\mathrm{A}_{3}\right) \lambda \beta e^{-d_{2} \tau}\left(q^{2} /\left(c+q^{2}\right)\right)-d_{3}-M d_{4}>0, \\
& \left(\mathrm{~A}_{4}\right) 1-p_{1}>e^{\left((\beta M / \lambda c) m_{2}^{*}+d_{1}\right) T} /(1+r),
\end{aligned}
$$

where $M, m_{2}^{*}$, and $q$ are defined in (14), (34), and (39), respectively.

Proof. It is obvious that the third equation of system (4) can be rewritten as follows:

$$
\begin{aligned}
\dot{y}_{2}(t)= & \left(\lambda \beta e^{-d_{2} \tau} \frac{x^{2}(t)}{c+x^{2}(t)}-d_{3}-d_{4} y_{2}(t)\right) y_{2}(t) \\
& -\lambda \beta e^{-d_{2} \tau} \frac{d}{d t} \int_{t-\tau}^{t} \frac{x^{2}(\theta)}{c+x^{2}(\theta)} y_{2}(\theta) d \theta .
\end{aligned}
$$

Define $V(t)=y_{2}(t)+\lambda \beta e^{-d_{2} \tau} \int_{t-\tau}^{t}\left(x^{2}(\theta) /\left(c+x^{2}(\theta)\right)\right) y_{2}(\theta) d \theta$.

By computation, we have

$$
\dot{V}(t)=\left(\lambda \beta e^{-d_{2} \tau} \frac{x^{2}(t)}{c+x^{2}(t)}-d_{3}-d_{4} y_{2}(t)\right) y_{2}(t) .
$$

Applying Lemma 4, we have

$$
\dot{V}(t)>\left(\lambda \beta e^{-d_{2} \tau} \frac{x^{2}(t)}{c+x^{2}(t)}-d_{3}-M d_{4}\right) y_{2}(t) .
$$

By hypothesis $\left(\mathrm{A}_{3}\right)$, for the arbitrary small constant $\varepsilon_{4}>0$, we have

$$
\lambda \beta e^{-d_{2} \tau} \frac{\left(q+\varepsilon_{4}\right)^{2}}{c+\left(q+\varepsilon_{4}\right)^{2}}>d_{3}+M d_{4} .
$$

Let $m_{2}^{*}$ be determined by the following equation:

$$
\frac{1+r}{r} K-\frac{e^{\left(\beta(M / c \lambda) m_{2}^{*}+d_{1}\right) T}}{\left(1-p_{1}\right) r} K=\sqrt{\frac{c\left(d_{3}+M d_{4}\right)}{\lambda \beta e^{-d_{2} \tau}-d_{3}-M d_{4}}} .
$$

Then, for any $t_{4}>0$, it is impossible that $y_{2}(t)<m_{2}^{*}$ for all $t>t_{4}$. Suppose that the claim is invalid; then, there exists $t_{4}>0$ such that $y_{2}(t)<m_{2}^{*}$ for all $t>t_{4}$. It follows from the first equation of system (4) that

$$
\begin{aligned}
\dot{x}(t) & \geq-\left(\beta \frac{M}{c \lambda} m_{2}^{*}+d_{1}\right) x(t), \quad t \neq(n+l-1) T, \quad t \neq n T, \\
x\left(t^{+}\right) & =x(t)+r x(t)\left(1-\frac{x(t)}{K}\right), \quad t=(n+l-1) T, \\
x\left(t^{+}\right) & =\left(1-p_{1}\right) x(t), \quad t=n T,
\end{aligned}
$$

for all $t>t_{4}$. Consider the auxiliary system of (35) as follows:

$$
\begin{gathered}
\dot{z}_{5}(t)=-\left(\beta \frac{M}{\lambda c} m_{2}^{*}+d_{1}\right) z_{5}(t), \quad t \neq(n+l-1) T, t \neq n T, \\
z_{5}\left(t^{+}\right)=z_{5}(t)+r z_{5}(t)\left(1-\frac{z_{5}(t)}{K}\right), \quad t=(n+l-1) T, \\
z_{5}\left(t^{+}\right)=\left(1-p_{1}\right) z_{5}(t), \quad t=n T .
\end{gathered}
$$

By hypothesis $\left(\mathrm{A}_{4}\right)$, we can obtain from Lemma 3 that

$$
z_{5}^{*}(t)=\left\{\begin{array}{c}
e^{\left(\beta(M / c \lambda) m_{2}^{*}+d_{1}\right) l T}\left(\frac{1+r}{r} K-\frac{e^{\left(\beta(M / c \lambda) m_{2}^{*}+d_{1}\right) T}}{\left(1-p_{1}\right) r} K\right) \\
\times e^{-\left(\beta(M / c \lambda) m_{2}^{*}+d_{1}\right)(t-(n-1) T)}, \\
t \in((n-1) T,(n+l-1) T], \\
\frac{e^{\left(\beta(M / c \lambda) m_{2}^{*}+d_{1}\right) T}}{1-p_{1}}\left(\frac{1+r}{r} K-\frac{e^{\left(\beta(M / c \lambda) m_{2}^{*}+d_{1}\right) T}}{\left(1-p_{1}\right) r} K\right) \\
\times e^{-\left(\beta(M / c \lambda) m_{2}^{*}+d_{1}\right)(t-(n+l-1) T)}, \\
t \in((n+l-1) T, n T],
\end{array}\right.
$$

which is the unique positive periodic solution of system (36) and is globally asymptotically stable. By the comparison theorem of impulsive differential equation, we know there exists $t_{5}\left(>t_{4}+\tau\right)$ such that $x(t) \geq z_{5}^{*}(t)-\varepsilon_{4}$.

On the other hand, for all $t \geq t_{5}$, we have

$$
\begin{aligned}
z_{5}^{*}(t) & \geq\left\{\begin{array}{c}
\frac{1+r}{r} K-\frac{e^{\left(\beta(M / c \lambda) m_{2}^{*}+d_{1}\right) T}}{\left(1-p_{1}\right) r} K \\
\left(\frac{1+r}{r} K-\frac{e^{\left(\beta(M / c \lambda) m_{2}^{*}+d_{1}\right) T}}{\left(1-p_{1}\right) r} K\right) \frac{e^{\left(\beta(M / c \lambda) m_{2}^{*}+d_{1}\right) l T}}{\left(1-p_{1}\right)}
\end{array}\right\} \\
\geq & \frac{1+r}{r} K-\frac{e^{\left(\beta(M / c \lambda) m_{2}^{*}+d_{1}\right) T}}{\left(1-p_{1}\right) r} K \stackrel{\Delta}{=} \sigma .
\end{aligned}
$$

Thus,

$$
x(t) \geq \sigma-\varepsilon_{4} \stackrel{\Delta}{=} q, \quad \forall t \geq t_{5} .
$$

By (33), we have

$$
\lambda \beta e^{-d_{2} \tau} \frac{\sigma^{2}}{c+\sigma^{2}}>d_{3}+M d_{4} .
$$

Hence, by (32) and (40), we have

$$
\dot{V}(t) \geq\left(\lambda \beta e^{-d_{2} \tau} \frac{\sigma^{2}}{c+\sigma^{2}}-d_{3}-M d_{4}\right) y_{2}(t), \quad t \geq t_{5} .
$$

Let $y_{2}^{m}=\min _{t \in\left[t_{1}, t_{1}+\tau\right]} y_{2}(t)$. We will show that $y_{2}(t) \geq y_{2}^{m}$ for all $t \geq t_{5}$. Otherwise, there exists a constant $\alpha>0$ such that $y_{2}(t) \geq y_{2}^{m}$ for $t_{5} \leq t \leq t_{5}+\tau+\alpha, y_{2}\left(t_{5}+\tau+\alpha\right) \geq y_{2}^{m}$, and $\dot{y}_{2}\left(t_{5}+\tau+\alpha\right)<0$. However, by the third equation of system (4) and (40), we have

$$
\begin{array}{r}
\dot{y}_{2}\left(t_{5}+\tau+\alpha\right) \geq\left(\lambda \beta e^{-d_{2} \tau} \frac{\sigma^{2}}{c+\sigma^{2}}-d_{3}-M d_{4}\right) y_{2}^{m}>0, \\
t \geq t_{5},
\end{array}
$$




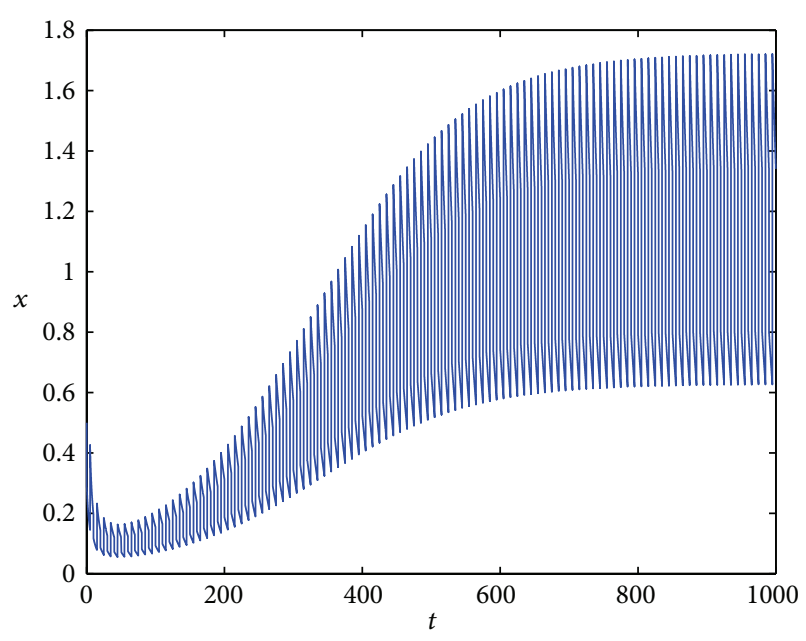

(a)

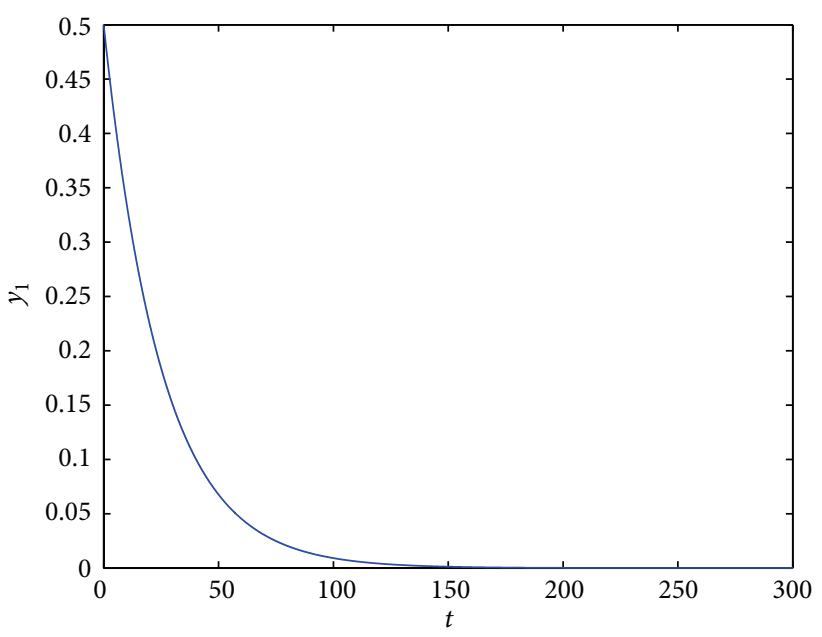

(b)

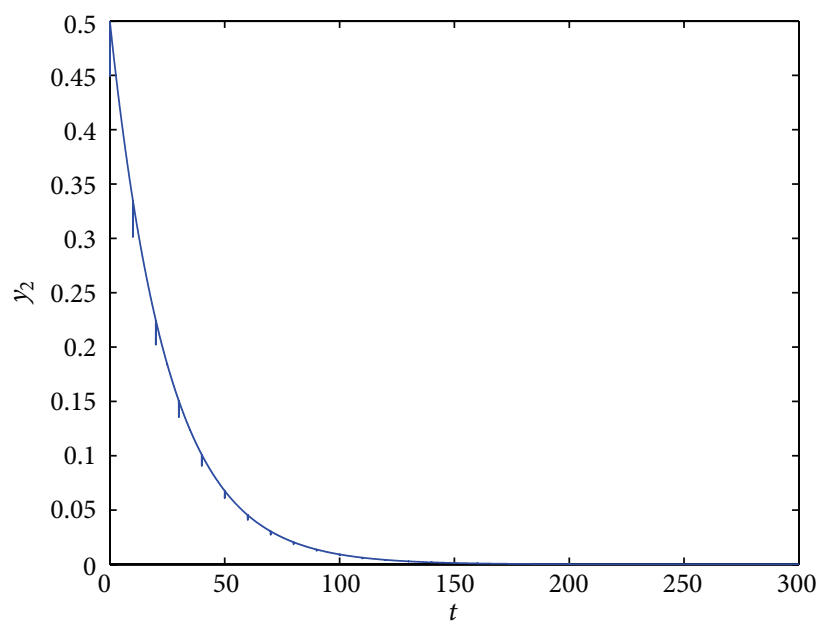

(c)

Figure 1: Dynamical behavior of system (4) with $r=2, K=5, \beta=1, c=10, d_{1}=0.5, \lambda=1, d_{2}=0.4, d_{3}=0.3, d_{4}=0.1, p_{1}=0.4$, $p_{2}=0.1, \tau=0, T=1$. (a) Time series of the prey population; (b) time series of the immature predator population; (c) time series of the mature predator population.

which is a contradiction. Thus, we have $y_{2}(t) \geq y_{2}^{m}$ for all $t \geq t_{5}$.

By (40) and (41), therefore,

$$
\dot{V}(t)>\left(\lambda \beta e^{-d_{2} \tau} \frac{\sigma^{2}}{c+\sigma^{2}}-d_{3}-M d_{4}\right) y_{2}^{m}>0, \quad t \geq t_{5},
$$

which means that $V(t) \rightarrow \infty$ as $t \rightarrow \infty$. It is a contradiction with $V(t) \leq(M / \lambda)\left(1+r \tau e^{-d_{1} \tau}\right)$. Therefore, for any $t_{4}>0$, the inequality $y_{2}(t)<m_{2}^{*}$ cannot hold for all $t \geq t_{4}$. So, there exist the following two cases:

(i) if $y_{2}(t) \geq m_{2}^{*}$ holds for all $t$ large enough, then our goal is obtained;

(ii) if $y_{2}(t)$ is oscillatory about $m_{2}^{*}$, let

$$
m_{2}=\min \left\{\frac{m_{2}^{*}}{2}, m_{2}^{*} e^{-\left(d_{3}+d_{4} m_{2}^{*}\right) \tau}\right\} .
$$

We prove that $y_{2}(t) \geq m_{2}$ for all $t$ sufficiently large. Suppose that there exist positive constants $\mu, \eta$ such that $y_{2}(\mu)=$ $y_{2}(\mu+\eta)=m_{2}^{*}$ and $y_{2}(t)<m_{2}^{*}$ for all $\mu<t<\mu+\eta$, and inequality (40) holds true for $\mu<t<\mu+\eta$, where $\mu$ is sufficiently large enough. Since $y_{2}(t)$ is continuous and bounded and is not affected by impulses, we conclude that $y_{2}(t)$ is uniformly continuous. Hence, there exists a constant $T_{0}\left(0<T_{0}<\tau\right.$ and $T_{0}$ is independent of the choice of $\left.\mu\right)$ such that $y_{2}(\mu)>m_{2}^{*} / 2$ for $\mu \leq t \leq \mu+T_{0}$. If $\eta \leq T_{0}$, our aim is obtained. If $T_{0}<\eta \leq \tau$, from the third equation of (4), we have that, for $\mu<t<\mu+\eta, \dot{y}_{2}(t) \geq-d_{3} y_{2}(t)-d_{4} y_{2}^{2}(t)$. According to the assumptions $y_{2}(\mu)=m_{2}^{*}$ and $y_{2}(\mu)<m_{2}^{*}$ for $\mu<t<\mu+\eta$, we have $\dot{y}_{2}(t) \geq-\left(d_{3}+d_{4} m_{2}^{*}\right) y_{2}(t)$ for $\mu<$ $t \leq \mu+\eta \leq \mu+\tau$. Then, we derive that $y_{2}(t) \geq m_{2}^{*} e^{-\left(d_{3}+d_{4} m_{2}^{*}\right) \tau}$. It is clear that $y_{2}(t) \geq m_{2}$ for $\mu \leq t \leq \mu+\eta$. If $\eta \geq \tau$, then we have $y_{2}(t) \geq m_{2}$ for $\mu<t \leq \mu+\tau$. The same arguments can be continued. We can obtain $y_{2}(t) \geq m_{2}$ for $\mu+\tau \leq t \leq \mu+\eta$. Since the interval $[\mu, \mu+\eta]$ is arbitrarily chosen, we get that 


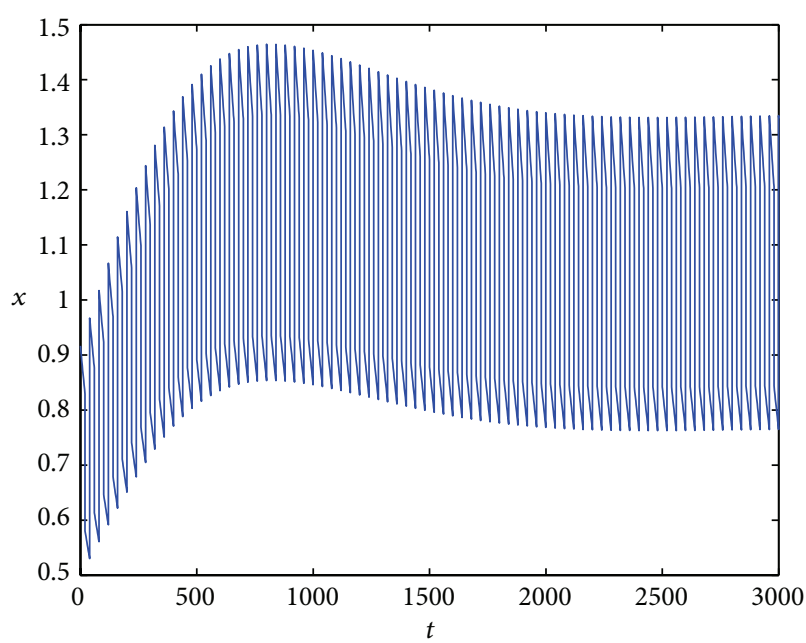

(a)

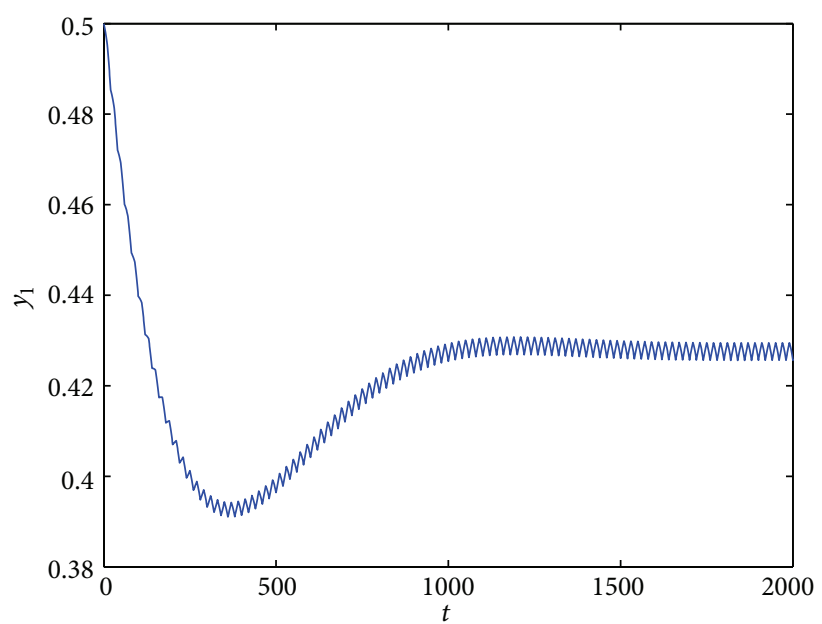

(b)

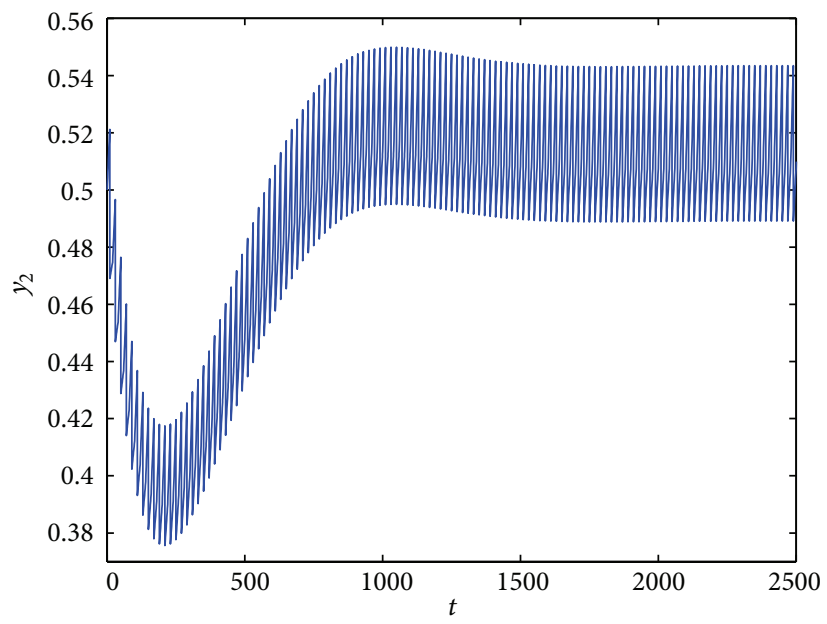

(c)

Figure 2: Dynamical behavior of system (4) with $r=1, K=3, \beta=2, c=1, d_{1}=0.5, \lambda=1, d_{2}=0.3, d_{3}=0.2, d_{4}=0.1, p_{1}=0.3, p_{2}=0.1$, $\tau=1, T=0.1$. (a) Time series of the prey population; (b) time series of the immature predator population; (c) time series of the mature predator population.

$y_{2}(t) \geq m_{2}$ for $t$ sufficiently large. In view of our arguments above, the choice of $m_{2}$ is independent of the positive solution of (4) which satisfies that $y_{2}(t) \geq m_{2}$ for $t$ large enough.

Next, from the second equation of system (4), we have

$$
\dot{y}_{1}(t) \geq \lambda \beta m_{2}\left(1-e^{-d_{2} \tau}\right)-d_{2} y_{1}(t) \text {. }
$$

Integrating (45) and using comparison theorem, we can easily get

$$
y_{1}(t) \geq \min \left\{y_{1}\left(0^{+}\right), \frac{\lambda \beta m_{2}\left(1-e^{-d_{2} \tau}\right)}{d_{2}}\right\} \triangleq m_{1} .
$$

On the other hand, by (29), we have $x(t) \geq x^{*}(t)-\varepsilon_{1} \stackrel{\Delta}{=} m_{0}$. Let $m=\min \left\{m_{0}, m_{1}, m_{2}\right\}$; then, we have $x(t) \geq m, y_{1}(t) \geq m$, $y_{2}(t) \geq m$. By Lemma 4 and above discussion, system (4) is permanent. This completes the proof.

\section{Examples and Numerical Simulations}

In this section, we give some examples and numerical simulations to show the effectiveness of the main results.

In system (4), we let $r=2, K=5, \beta=1, c=10, d_{1}=0.5$, $\lambda=1, d_{2}=0.4, d_{3}=0.3, d_{4}=0.1, p_{1}=0.4, p_{2}=0.1, \tau=0$, $T=1$. By computation, one can obtain that the conditions of Theorem 6 are satisfied, so the predator-extinction periodic solution is globally attractive, which can be shown by Figure 1 . If $r=1, K=3, \beta=2, c=1, d_{1}=0.5, \lambda=1, d_{2}=0.3$, $d_{3}=0.2, d_{4}=0.1, p_{1}=0.3, p_{2}=0.1, \tau=1, T=0.1$, then by computation, the conditions of Theorem 8 are also satisfied; hence, by Theorem 8, system (4) is permanent; see Figure 2.

\section{Conclusion}

In this paper, a stage-structured predator-prey model with Holling type III functional response, birth pulse, and impulsive harvesting at different moments is proposed. By using 
comparison theorem of impulsive differential equations and some analysis techniques, the sufficient conditions ensuring the predator-extinction periodic solution and the permanence of system (4) are obtained. Theorem 6 implies that reducing $p_{1}$ and $T$ appropriately and $\left(A_{2}\right)$ can be propitious to the global attractivity of the predator-extinction periodic solution $\left(x^{*}(t), 0,0\right)$. That is, if the mature predator is caught excessively or the immature predator is stocked too few, it will lose the merits of exploitative mature predator population. Similarly, we can see from Theorem 8 that reducing $p_{1}$ and $T$ appropriately and $\left(\mathrm{A}_{4}\right)$ are in favor of the permanence of system (4). It implies that reasonable harvesting on mature predator and appropriate protecting on immature predator play an important role in the permanence of system (4). Moreover, by Theorems 6 and 8, we believe that there exists a sharp threshold, which is beneficial for people to make the best use of biological resources but will not break the biological balance. It will be interesting for us to continue to study the optimal harvesting policy of system (4) in the near future.

\section{Conflict of Interests}

The authors declare that there is no conflict of interests regarding the publication of this paper.

\section{Acknowledgments}

This paper is supported by National Natural Science Foundation of China $(11161015,11361012)$ and Natural Science Foundation of Guangxi (2013GXNSFAA019003) and partially supported by the National High Technology Research and Development Program 863 under Grant no. 2013AA12A402.

\section{References}

[1] W. X. Li and K. Wang, "Optimal harvesting policy for general stochastic logistic population model," Journal of Mathematical Analysis and Applications, vol. 368, no. 2, pp. 420-428, 2010.

[2] T. Das, R. N. Mukherjee, and K. S. Chaudhuri, "Harvesting of a prey-predator fishery in the presence of toxicity," Applied Mathematical Modelling, vol. 33, no. 5, pp. 2282-2292, 2009.

[3] R. Zhang, J. F. Sun, and H. X. Yang, "Analysis of a prey-predator fishery model with prey reserve," Applied Mathematical Sciences, vol. 1, no. 50, pp. 2481-2492, 2007.

[4] Z. J. Zeng, "Asymptotically periodic solution and optimal harvesting policy for gompertz system," Nonlinear Analysis: Real World Applications, vol. 12, no. 3, pp. 1401-1409, 2011.

[5] X. Y. Song and L. S. Chen, "Optimal harvesting and stability for a two-species competitive system with stage structure," Mathematical Biosciences, vol. 170, no. 2, pp. 173-186, 2001.

[6] T. K. Kar, "Selective harvesting in a prey-predator fishery with time delay," Mathematical and Computer Modelling, vol. 38, no. 3-4, pp. 449-458, 2003.

[7] X. W. Jiang, Q. Song, and M. Y. Hao, "Dynamics behaviors of a delayed stage-structured predator-prey model with impulsive effect," Applied Mathematics and Computation, vol. 215, no. 12, pp. 4221-4229, 2010.
[8] J. J. Jiao, X. Z. Meng, and L. S. Chen, "A stage-structured Holling mass defence predator-prey model with impulsive perturbations on predators," Applied Mathematics and Computation, vol. 189, no. 2, pp. 1448-1458, 2007.

[9] Z. J. Liu and R. H. Tan, "Impulsive harvesting and stocking in a Monod-Haldane functional response predator-prey system," Chaos, Solitons and Fractals, vol. 34, no. 2, pp. 454-464, 2007.

[10] Y. Shao and B. Dai, "The dynamics of an impulsive delay predator-prey model with stage structure and Beddington-type functional response," Nonlinear Analysis: Real World Applications, vol. 11, no. 5, pp. 3567-3576, 2010.

[11] X. Song, M. Hao, and X. Meng, "A stage-structured predatorprey model with disturbing pulse and time delays," Applied Mathematical Modelling, vol. 33, no. 1, pp. 211-223, 2009.

[12] Y. F. Shao and Y. Li, "Dynamical analysis of a stage structured predator-prey system with impulsive diffusion and generic functional response," Applied Mathematics and Computation, vol. 220, pp. 472-481, 2013.

[13] F. Y. Wei and K. Wang, "Persistence of some stage structured ecosystems with finite and infinite delay," Nonlinear Analysis: Real World Application, vol. 12, pp. 1401-1409, 2011.

[14] Z. Y. Xiang, D. Long, and X. Y. Song, "A delayed Lotka-Volterra model with birth pulse and impulsive effect at different moment on the prey," Applied Mathematics and Computation, vol. 219, no. 20, pp. 10263-10270, 2013.

[15] J. Jiao, S. Cai, and L. Chen, "Analysis of a stage-structured predatory-prey system with birth pulse and impulsive harvesting at different moments," Nonlinear Analysis. Real World Applications, vol. 12, no. 4, pp. 2232-2244, 2011.

[16] Z. Ma, J. Yang, and G. Jiang, "Impulsive control in a stage structure population model with birth pulses," Applied Mathematics and Computation, vol. 217, no. 7, pp. 3453-3460, 2010.

[17] J. Liu, "Analysis of an epidemic model with density-dependent birth rate, birth pulses," Communications in Nonlinear Science and Numerical Simulation, vol. 15, no. 11, pp. 3568-3576, 2010.

[18] C. Zhang, N.-J. Huang, and D. O'Regan, “Almost periodic solutions for a Volterra model with mutual interference and Holling type III functional response," Applied Mathematics and Computation, vol. 225, pp. 503-511, 2013.

[19] Y. F. Lv, R. Yuan, and Y. Z. Pei, "A prey-predator model with harvesting for fishery resource with reserve area," Applied Mathematical Modelling, vol. 37, no. 5, pp. 3048-3062, 2013.

[20] B. Shulgin, L. Stone, and Z. Agur, "Pulse vaccination strategy in the SIR epidemic model," Bulletin of Mathematical Biology, vol. 60, no. 6, pp. 1123-1148, 1998.

[21] V. Lakshmikantham, D. D. Bainov, and P. S. Simeonov, Theory of Impulsive Differential Equations, World Scientific, Singapore, 1989. 


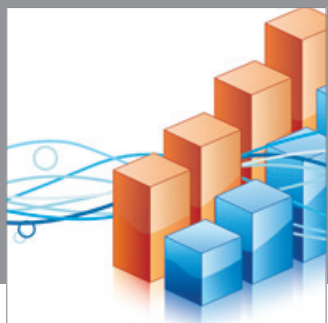

Advances in

Operations Research

mansans

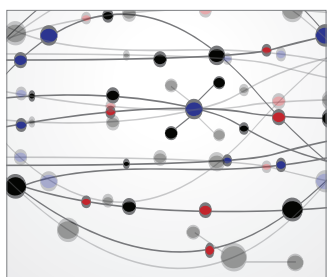

The Scientific World Journal
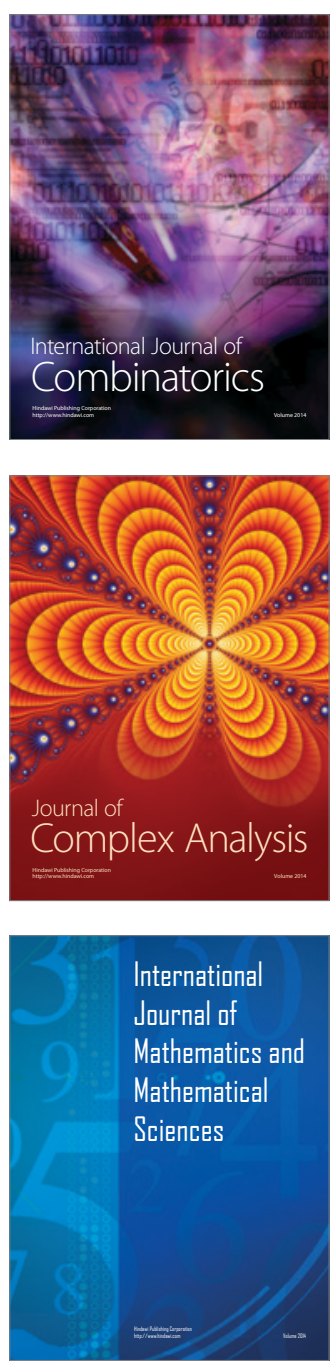
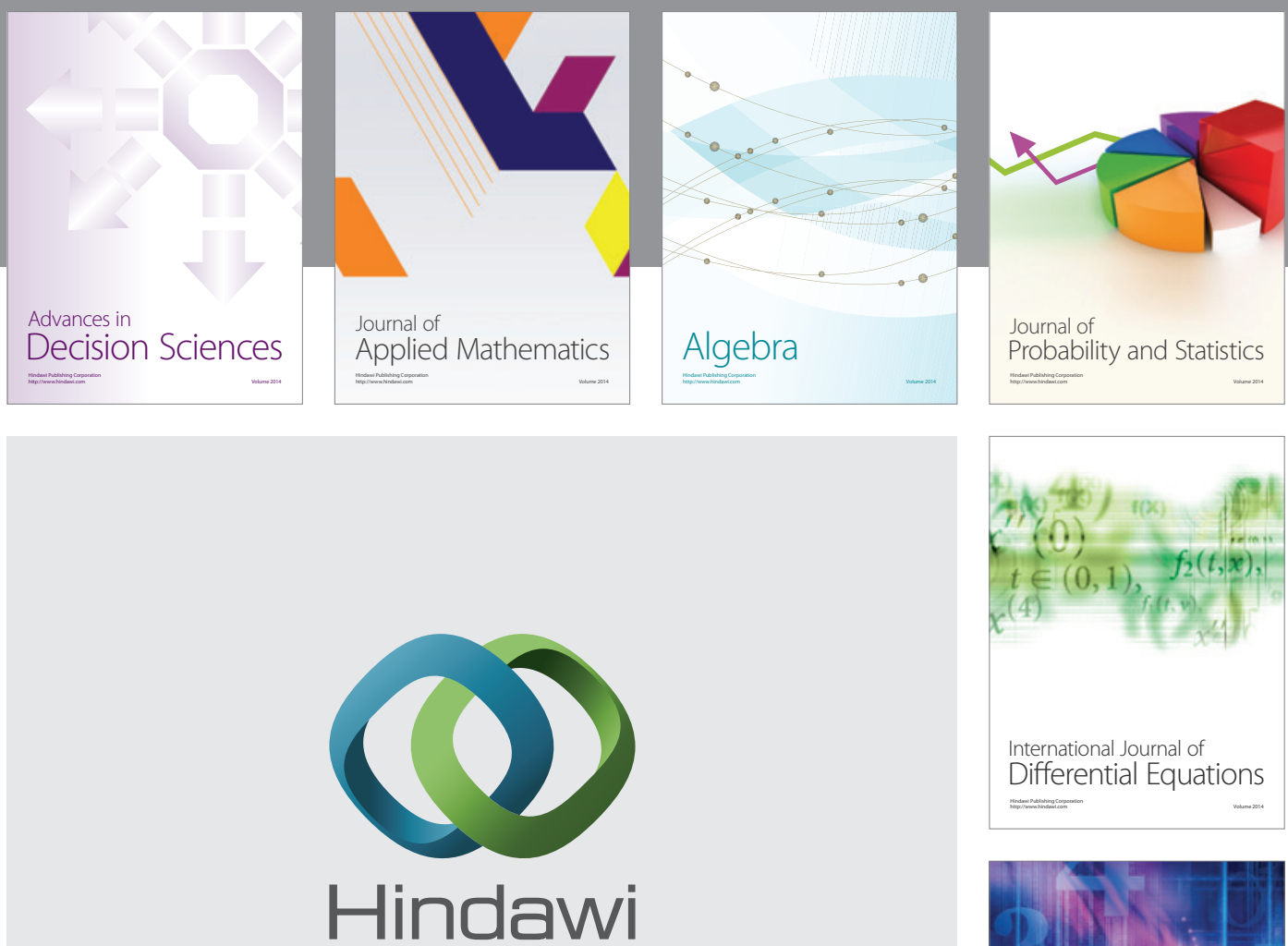

Submit your manuscripts at http://www.hindawi.com
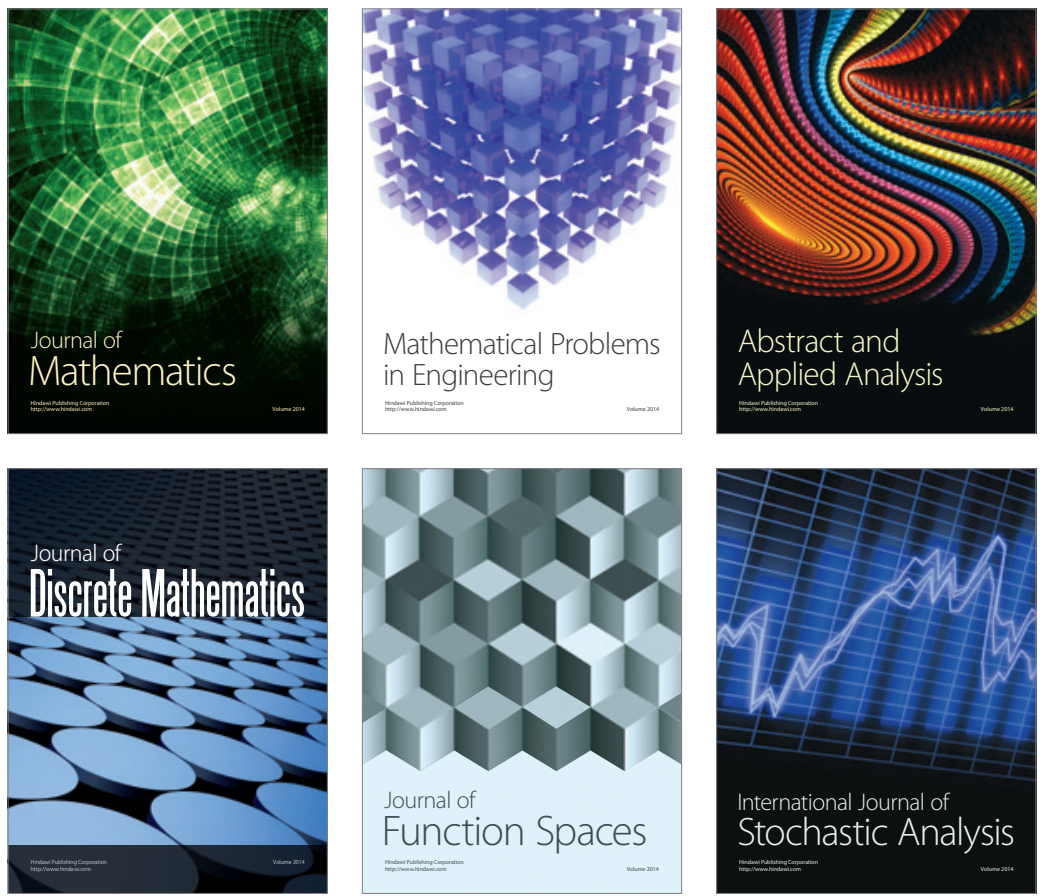

Journal of

Function Spaces

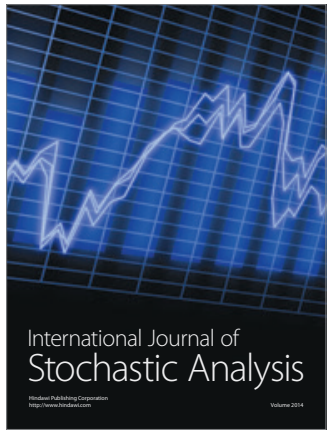

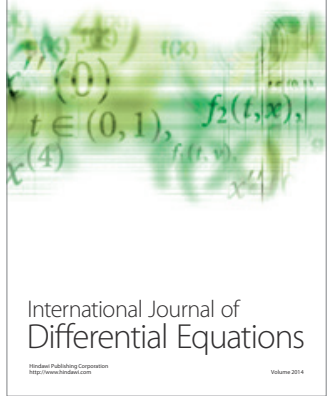
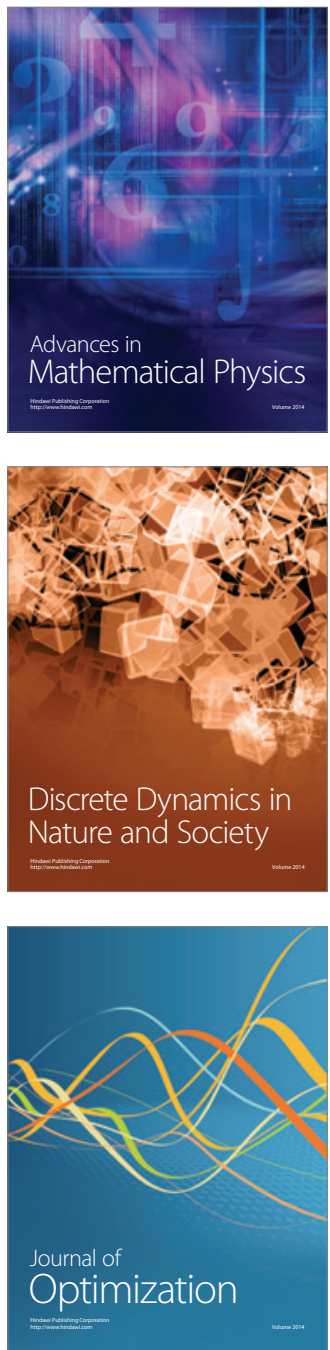